\title{
ESTRATEGIAS PARA LA EXPANSIÓN Y DESARROLLO ECONÓMICO PARA EL RESTAURANTE CUETSAFOOD DE COMIDA SALUDABLE Y SOSTENIBLE EN BOGOTÁ
}

Expansion and economic development strategies for Cuetsafood restaurant (healthy and sustainable food) in Bogotá

\author{
SARAH NATALIA WILCHES GÓMEZ \\ swilches08@unisalle.edu.co \\ DIANA MARÍA VALENZUELA SOLANO \\ dvalenzuela31@unisalle.edu.co \\ JACKSON PAUL PEREIRA SILVA (Docente investigador) \\ jacpereira@unisalle.edu.co \\ Semillero de investigación SeEmprende \\ Universidad de La Salle (Colombia)
}

Artículo de investigación formativa

Recepción: 20 de septiembre de 2020

Aceptación: 17 de febrero de 2021 


\section{Resumen:}

El objetivo de la investigación es posicionar al restaurante Cuetsafood a través de una planeación estratégica de marketing; la idea es compartir una gran producción encargada de generar un cambio alimentario y ecológico. Así, se presenta la oportunidad de sostener una alimentación saludable y costearlo; CUETSA ofrece la ocasión para que los consumidores puedan acceder una comida orgánica y balanceada a cambio de un precio justo. Allí se puede disfrutar con seguridad que el esfuerzo económico es razonable respecto a la calidad del producto entregado. El artículo presenta una metodología de recolección de información de carácter cualitativo; por medio de la literatura revisada se pretende describir una situación en la que se explique el comportamiento de los individuos respecto al consumo de alimentos saludables y sostenibles. También es de tipología aplicada tecnológica pues identifica el conocimiento del sector productivo de bienes y servicios (restaurante y alimentos) para mejorarlo y hacerlo más eficiente.

Palabras clave: desarrollo sostenible; mercadeo; comportamiento del consumidor; cocina.

\section{Abstract:}

The purpose of the research is to stand "Cuetsafood" restaurant through strategic marketing planning; the idea is to share a large production in charge of generating a nutritional and ecological change. Thus, there is the opportunity to sustain and afford a healthy diet; CUETSA offers the opportunity for consumers to have access to an organic and balanced meal in exchange for a fair price. There, you can safely enjoy that the economic effort is reasonable regarding to the quality of the delivered product. The article presents a qualitative information gathering methodology; through the literature reviewed, it is intended to describe a situation in which the behavior of individuals regarding the consumption of healthy and sustainable foods is explained. It is also composed of applied technological typology since it identifies the knowledge of the productive sector of goods and services (restaurant and food) to improve it and make it more efficient.

Keywords: sustainable development; marketing; consumers behavior; cuisine. 


\section{Introducción}

Sin importar su edad, los individuos deben aspirar a una mejor calidad de vida a medida que envejecen. Para esto, necesitan el acceso a un tipo de mercado más natural, orgánico y sostenible en cuanto a cualquier bien o servicio, en este caso específico la alimentación. Sin embargo, las personas no suelen invertir en ese mercado lo que significa que la demanda en el consumo de alimentos saludables es escasa. También ocasiona que los individuos no consideren dicha clase de práctica alimentaria como indispensable para su salud. A esto se le suma que, aunque quisieran, adquirirlos es complejo por su elevado costo.

El problema radica en que en la modernidad la forma de consumo de este tipo de alimentos no es constituido como parte de sus costumbres. Lo que antes se consideraba simple o desabrido, ahora es apreciado como exclusivo. En consecuencia, la exclusividad desarrollada transforma el hecho de que adquirir ese tipo de víveres sea un privilegio y no una necesidad.

Cuetsafood (CUESTA) brinda y representa un modo innovador de alimentación donde promueve prácticas saludables para el ser humano; asimismo, son amigables con el medio ambiente. El restaurante considera que el concepto de comida hace parte importante de dinámicas sociales y culturales; aquellas utilizadas para interactuar y compartir experiencias; de esta manera, conformando una identidad que define a cada individuo. Por lo anterior, se pretende demostrar que las personas pueden tener fácil acceso a este tipo de alimentos y que gracias a CUETSA es posible ser parte de una gran experiencia gastronómica.

\section{Metodología}

A partir del objetivo planteado para la investigación se ha diseñado una encuesta que responda ante las preferencias y comportamientos del consumidor, respecto a los restaurantes a puerta cerrada y alimentación saludable. El propósito es identificar el segmento de tipos de clientes y sus tendencias de consumo a través de sus experiencias.

\section{Entrevista a clientes.}

\section{EDAD:}

1.Teniendo en cuenta la coyuntura actual, ¿prefiere dirigirse al restaurante o pedir domicilio?

2. ¿Cuenta usted con la facilidad de pedir comida a domicilio frecuentemente?

3.Cuando se dirige a restaurantes o pide por domicilio, ¿presta atención al tipo de comida que consume? ¿Prefiere comida poco o muy saludable?

4.¿Qué tanto conoce sobre la comida saludable? ¿Prefiere prepararla en casa o adquirirla en restaurantes? 
5.¿Qué tipos de platos le gustaría ver en el menú de un restaurante? ¿Le gustaría platos tradicionales o sofisticados?

6. ¿Ha escuchado sobre el concepto de "restaurante a puerta cerrada"? ¿Qué interpreta del concepto?

7. ¿Usted cree que un restaurante a puerta cerrada permite espacios familiares?

8. ¿Se cohibiría por algún motivo a pedir comida saludable en un restaurante a puerta cerrada?

9. ¿Cuáles son los factores determinantes que revisa antes de ir a un nuevo restaurante? (Recomendaciones en internet, calificaciones, opiniones de conocidos, entre otros).

10.¿Por cuál tipo de aplicación prefiere recibir información sobre el menú, eventos y servicios? Facebook, Instagram, Twitter, otro.

\section{Resultados}

Las costumbres de los individuos en su manera de conseguir el alimento ha evolucionado dependiendo de su adaptación al entorno; hemos evidenciado como la industria de alimentos ha simplificado y transformado los comestibles en productos manipulados para facilitar su consumo y ha hecho de estos un proceso menos saludable.

Para las generaciones actuales, ha sido posible contar con acceso a una amplia información sobre lo fundamental que es para los individuos mantener una alimentación balanceada. Jiménez, Vivas, Guzmán, Díaz y Reyes (2016), sustentan que "la gastronomía ha estado generando diferentes desafíos, ello como resultado de una mayor especialización por parte de los consumidores, quienes van más allá de la manipulación de alimentos, exigiendo una mayor calidad en el producto y en el servicio ofrecido" (p. 2).

Se encuentra que para lograr ampliar la participación de CUETSA en el sector gastronómico de la ciudad de Bogotá, es necesario que a través de las generaciones jóvenes sean fomentadas prácticas de consumo de alimentos balanceados enfatizando la importancia del cuidado de su salud.

\section{Conclusiones}

Esta investigación se fundamenta en la teoría del comportamiento del consumidor planteada por Thorndike y Skinner (1920) llamada "Teoría del Condicionamiento Operante" la cual define el comportamiento del individuo como una variable dependiente a estímulos ambientales o factores externos que tienen como respuesta elementos que pueden ser positivos o negativos y como consecuencia configuran las conductas del ser humano.

Basado en la anterior información, encontramos que en la ciudad de Bogotá podemos definir en grupos de edades como las personas a través de su cultura y generación, realizan un manejo particular del proceso de alimentación en sus hogares. Es por esto que debe ser considerado continuar promoviendo practicas provechosas para los restaurantes y asi aumentar la demanda de alimentos saludables dentro del sector gastronómico de la ciudad de Bogotá. 


\section{Referencias bibliográficas}

Jiménez, C., Reyes, J., Vivas, A., Guzmán, J., y Díaz, S. (2019). Planeamiento estratégico del sector gastronómico en Colombia (Tesis de maestría). Universidad Católica del Perú. Recuperado de http://tesis.pucp.edu.pe/repositorio/ handle/20.500.12404/14383 